\title{
Anticipating twin-twin transfusion syndrome in monochorionic twin pregnancy. Is there a role for nuchal translucency and ductus venosus blood flow evaluation at 11-14 weeks?
}

\author{
A Matias ${ }^{1}, \mathrm{~N}$ Montenegro ${ }^{1}$ and $\mathrm{JC}$ Areias $^{2}$ \\ ${ }^{1}$ Department of Obstetrics and Gynecology, Faculty of Medicine, Porto \\ ${ }^{2}$ Paediatric Cardiology Unit, Hospital of S João, Porto, Portugal
}

\begin{abstract}
Twin-twin transfusion syndrome is a major complication of monochorionic twin pregnancies. In foetuses from monochorionic twinning the presence of increased nuchal translucency thickness (NT) has been associated with an increased risk of developing this syndrome. One of the presumed mechanisms of increased NT is early cardiac failure, indirectly indicated by abnormal blood flow in the ductus venosus. We present eleven cases of monochorionic twin pregnancies in which nuchal translucency thickness and ductus venosus blood flow evaluation was performed at 11-14 weeks. In the two cases presenting with nuchal translucency discrepancy between the two foetuses along with anomalous ductus venosus blood flow in the foetus with increased nuchal translucency, twin-twin transfusion syndrome (TTTS) eventually developed. In none of the twins displaying no inter-twin difference in NT measurements and in those with discrepant NT but normal flow in both ductus venosus, was the progression to TTTS observed. In the two cases which developed TTTS, foetoscopic laser coagulation of the vascular anastomosis was successfully carried out at 18 weeks and normalisation of the venous return was registered. These findings suggest that the association of increased NT and abnormal flow in the ductus venosus in monochorionic twins may be an early manifestation of haemodynamic imbalance between the donor and the recipient eventually manifested as twin-twin transfusion syndrome. Further studies, how ever, are necessary to establish the potential role of the combination of NT and ductus venosus blood flow assessment as a screening method for TTTS. Twin Research (2000) 3, 65-70.
\end{abstract}

Keywords: monochorionic twins, nuchal translucency, ductus venosus blood flow, twin-twin transfusion syndrome

\section{Introduction}

In monochorionic pregnancies, increased NT at 10-14 weeks of gestation was detected twice as much as in singl eton pregnancies and was associated with a fourfold increase in the risk of developing twin-twin transfusion syndrome. ${ }^{1}$ Twin-twin transfusion syndrome (TTTS) is a severe complication in $4-26 \%$ of monochorionic twinning and accounts for up to $17 \%$ of perinatal mortality in twins. ${ }^{2}$

Recently we have provided evidence of heart failure by showing al terations in the ductus venosus blood flow in foetuses with increased nuchal translucency thickness (NT) and chromosomopathies ${ }^{3-5}$ and/or cardiac defects. ${ }^{6,7}$ Preliminary findings from our group showed that abnormal flow in the ductus venosus (DV) between 11-14 weeks in monochor-

Correspondence: Nuno Montenegro MD PhD, Ultrasound Unit, Department of Obstetrics and Gynecology, University Hospital of S Jaão, 4200 Porto, Portugal. Fax: 35125505870

Received 15 March 1999; revised 22 May 1999; accepted 4 July 1999 ionic twins with discrepant NT could be a precocious sign of circulatory imbal ance, later expressed as TTTS. $^{8}$

The aim of this study was to establish further the possible role of NT and DV blood flow evaluation at 11-14 weeks of gestation in anticipating TTTS manifested later in monochorionic twin pregnancies. Considering that in such twins there is no evidence of increased preval ence of chromosomopathies, both findings in the first trimester may be manifestations of haemodynamic disturbance associated with placental vascular communications and the early expression of heart failure.

\section{Material and methods}

In eleven pregnant women referred to our Ultrasound Unit for routine ultrasonographic assessment, monochorionic diamniotic pregnancy was identified between 11-14 weeks of gestation. In the first scan 
chorionicity and amnionicity were defined. Gestational age was calculated from crown-rump length. The foetal heart rate was within the normal range. ${ }^{9}$

NT thickness was measured and related to the crown-rump length $(2.2 \mathrm{~mm}$ at $38 \mathrm{~mm}$ increasing linearly to $2.8 \mathrm{~mm}$ at $84 \mathrm{~mm}$ ) to define the risk for chromosomal defects, as described el sewhere. ${ }^{10}$ As NT is increased in only $30 \%$ of the cases that will later develop TTTS, ${ }^{1}$ and unlikely to enhance significantly the prediction of TTTS, the inter-twin difference in NT measurements was set a cut-off at $0.7 \mathrm{~mm}$.

An ultrasound colour-coded Doppler machine (SSD2000, Aloka, Tokyo, Japan) with a 5-MHz transvaginal probe was used. The system operates at a maximum I spta (spatial peak temporal average) for pulsed Doppler mode of $92 \mathrm{~mW} / \mathrm{cm}^{2}$, well within the safety range approved. ${ }^{11,12}$ For the Doppler studies of the ductus venosus a right ventral mid-sagittal plane of the foetal trunk was first obtained during foetal quiescence and the pulsed Doppler gate was placed in the distal portion of the umbilical sinus; care was taken to avoid contamination from the intrahepatic portion of the umbilical vein, the left hepatic vein and inferior vena cava. ${ }^{13} \mathrm{An}$ average of five consecutive high quality waveforms was used to measure the peak velocity during ventricular systole (S-wave) and diastole (D-wave), the lowest forward velocity during atrial contraction in late diastole (A-wave) and the pulsatility index $(\mathrm{PI})$.

Doppler blood flow waveforms were al so obtained from the umbilical vein and umbilical artery. All pregnant women consented to participate in the study after being thoroughly informed about the aims and the still limited clinical information available.

A second scan was performed at about 17 weeks of gestation to detect any signs of TTTS. Severe TTTS was defined by the ultrasonographic features of anhydramnios and non-visible bladder in the donor foetus in combination with polyhydramnios and a dilated bladder in the recipient foetus. Doppler blood flow waveforms were recorded in the ductus venosus, umbilical vein and umbilical artery at the time of TTTS identification and after laser treatment every 2 weeks.

\section{Results}

The median maternal age was 31 (range 27-41) years and the median gestation was $12(12-13)$ weeks. In Table 1 the characteristics of the eleven cases are listed, with emphasis on NT measurement and presence, absence or reversal of A-wave in the ductus venosus and eventual development of TTTS.

In cases 1 and 3 NT discrepancy between the two twins was observed at 12 weeks. In the Doppler measurements no alterations were found except for the velocity during atrial contraction in the DV that was reversed in the foetus with increased NT and slightly reduced in the other one (Figure 1, case 3). A pulsatile pattern in the umbilical vein correlated with atrial contraction was recorded in the first foetus. End-diastolic blood flow was systematically present in the umbilical artery.

At 17 weeks of gestation ultrasonographic signs of TTTS appeared in cases 1 and 3 . In both cases discordance in the foetal size was found: the larger foetus had a distended bladder and was surrounded by polyhydramnios (presumed recipient), whereas the smaller twin (presumed donor) presented oligohydramnios and the bladder was not visible. No obvious foetal anomalies were detected. In the 'recipient' the velocity during atrial contraction in the DV was nearly zero and the umbilical artery PI was within the normal range. ${ }^{13}$ In the 'donor' the

Table 1 Parameters concerning the el even monochorionic twin pregnancies, including maternal age, gestational age at the time of the first examination, nuchal translucency thickness measured between 11-14 weeks, A-wave velocity in the ductus venosus (DV), the appearance of twin-twin transfusion syndrome (TTTS) and the type of treatment are discriminated

\begin{tabular}{|c|c|c|c|c|c|c|}
\hline Case & $\begin{array}{l}\text { Maternal } \\
\text { age } \\
\text { (years) }\end{array}$ & $\begin{array}{l}\text { Gestational } \\
\text { age } \\
\text { (weeks) }\end{array}$ & $\begin{array}{l}\text { Nuchal } \\
\text { translucency } \\
(\mathrm{mm})\end{array}$ & $\begin{array}{l}\text { DV } \\
\text { (A-wave) } \\
(\mathrm{cm} / \mathrm{s})\end{array}$ & TTTS & Treatment \\
\hline 1 & 28 & 12 & $3.3 / 3.7$ & $0 / \operatorname{Rev}$ & Yes & Laser \\
\hline 2 & 33 & 12 & $1.6 / 1.7$ & $\mathrm{~N} / \mathrm{N}$ & No & - \\
\hline 3 & 27 & 12 & $3.7 / 1.0$ & Rev/0 & Yes & Laser \\
\hline 4 & 41 & 12 & $1.5 / 1.0$ & $\mathrm{~N} / \mathrm{N}$ & No & - \\
\hline 5 & 28 & 12 & $1.5 / 1.3$ & $\mathrm{~N} / \mathrm{N}$ & No & - \\
\hline 6 & 31 & 12 & $0.7 / 0.8$ & $\mathrm{~N} / \mathrm{N}$ & No & - \\
\hline 7 & 40 & 12 & $1.7 / 1.7$ & $\mathrm{~N} / \mathrm{N}$ & No & - \\
\hline 8 & 36 & 13 & $2.7 / 1.4$ & $\mathrm{~N} / \mathrm{N}$ & No & - \\
\hline 9 & 23 & 12 & $1.0 / 1.0$ & $\mathrm{~N} / \mathrm{N}$ & No & - \\
\hline 10 & 30 & 12 & $1.1 / 1.0$ & $\mathrm{~N} / \mathrm{N}$ & No & - \\
\hline 11 & 33 & 10 & $1.0 / 1.0$ & $\mathrm{~N} / \mathrm{N}$ & No & - \\
\hline
\end{tabular}

$\mathrm{N}=$ normal $; 0=$ absent flow during atrial systole; Rev = reverse flow during atrial contraction. 


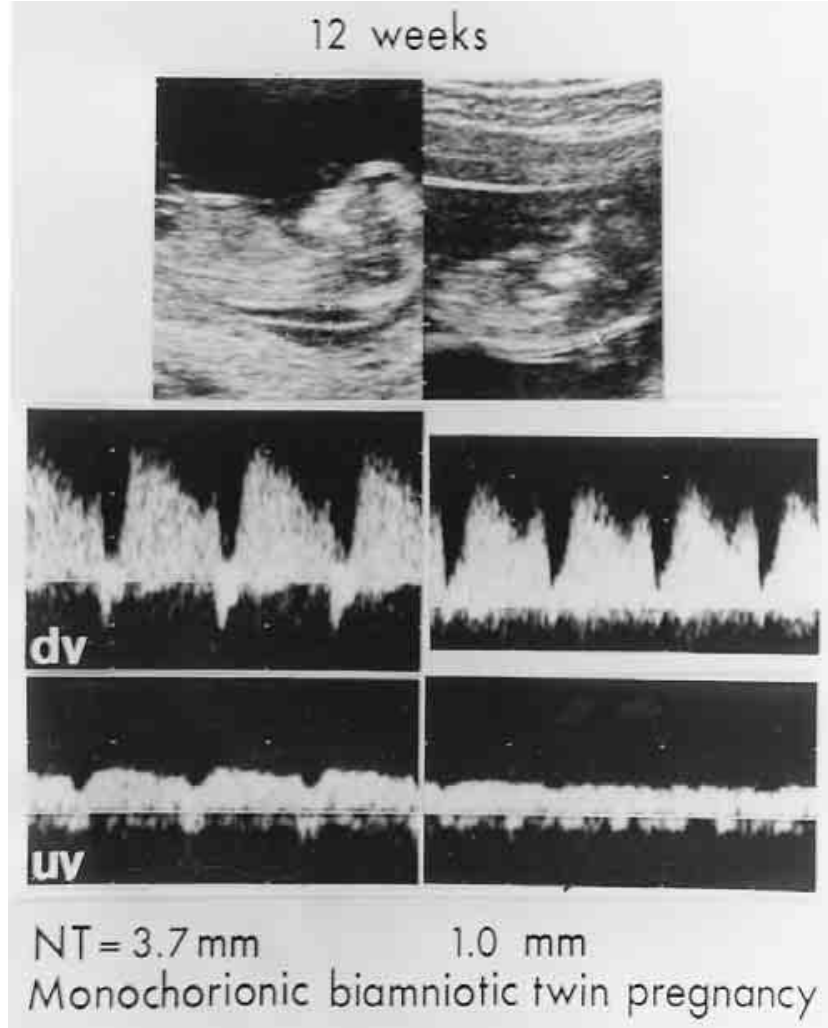

Figure 1 Doppler blood flow patterns in the umbilical vein (UV) and ductus venosus (DV) obtained in both foetuses at 12 weeks of gestation (case 3 ). In this examination a monochorionic biamniotic twin pregnancy was established. A nuchal translucency (NT) discrepancy was noted $(\mathrm{NT}=3.7 / 1.0 \mathrm{~mm})$

umbilical artery $\mathrm{PI}$ was increased and the AC velocity in the DV was diminished (Figure2, case 3 ).

Both patients were referred to the Harris Birthright Research Center for Fetal Medicine (London, UK) for foetoscopic laser separation of the vascular anastomosis at 17 weeks of gestation. Anti-D immunoglobulin was given and a Kleihauer test was performed in case 3 , as the patient was rhesus negative. One week later, the amniotic fluid around the 'donor' was recovered and the the bladder was visible in both cases. Both twins showed subsequently normal umbilical artery and DV blood flow waveforms (Figure 3, case 3), evaluated on a basis of every other two weeks.

In cases 2, 5, 6, 9, 10 and 11 NT measurements were similar for both twins. Doppler blood flow waveforms recorded in the ductus venosus at 12 weeks were normal in both twins and no signs of TTTS were observed later in gestation.

In cases 4 and 8 , al though a discrepancy in NT was detected between the two foetuses $(1.5 / 1.1 \mathrm{~mm}$ and $2.7 / 1.4 \mathrm{~mm}$, respectively) at 12-13weeks of gestation, the blood flow pattern of the Doppler waveform in the ductus venosus was normal for both twins.

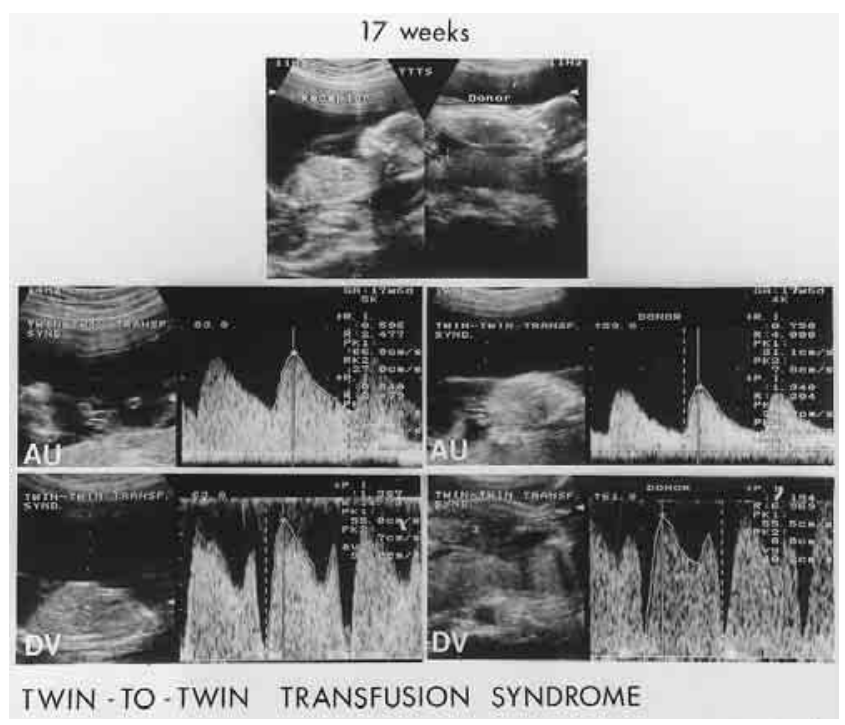

Figure 2 Doppler blood flow patterns in the umbilical artery (AU) and ductus venosus (DV) obtained in both foetuses at 17 weeks of gestation (case 3 ), when twin-twin transfusion was detected. Note the 'stuck twin' with oligohydramnios (donor) and the polyhydramnios around the recipient

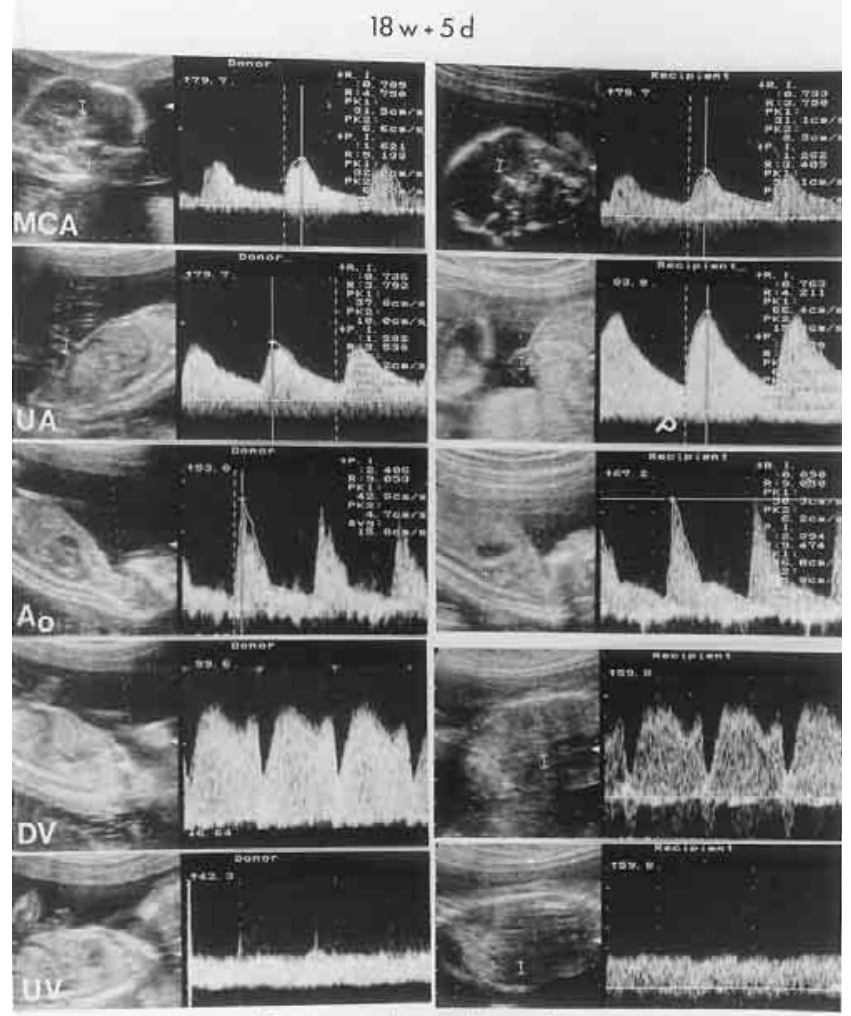

Twin-to-twin transfusion syndrome (post-laser)

Figure 3 Doppler blood flow profiles (arterial and venous) obtained in both foetuses (case 3 ) one week after foetoscopic laser coagulation. MCA: middle cerebral artery; UA: umbilical artery; Ao: descending aorta; DV: ductus venosus; UV: umbilical vein 
None of these foetuses devel oped signs of TTTS Iater in pregnancy.

The monochorionic diamniotic foetuses of our study were born alive and well; no major malformations were detected at birth, with the exception of case 3 . In this case, at 35 weeks one baby was found dead and a Cesarean section was performed. The other twin, a pale female baby, was delivered, weighing $1960 \mathrm{~g}$ and with an Apgar score of 6/8. The cord was entangled and the dead baby was pale.

As part of a continuing research project in which dichorionic pregnancies are evaluated for NT and blood flow in the DV, we selected an example of NT discrepancy in one of the dichorionic diamniotic twin pregnancies (Figure 4). In one of the foetuses (NT $=5.7 \mathrm{~mm}$ ) a reversed A-wave was recorded at 12 weeks. The foetus presenting normal NT (NT $=1.1 \mathrm{~mm}$ ) showed a normal DV blood flow pattern. Amniocentesis was performed and revealed a trisomy 21 and a chromosomally normal male foetus, respectively. The parents decided to proceed with the pregnancy.

\section{Discussion}

Twin-twin transfusion syndrome complicates $4 \%$ to $35 \%$ of monochorionic multiple pregnancies. These are characterised by the presence of vascular anastomoses between the two foetoplacental circulations, but a circulatory imbal ance resulting in the development of acute polyhydramnios/oligohydramnios in the second trimester of pregnancy occurs in hardly one third of the cases. ${ }^{2,14}$

Sebire and co-workers ${ }^{1}$ recently demonstrated a higher prevalence of increased NT among monochorionic pregnancies at 10-14 weeks of gestation and a fourfold increase in the risk of developing TTTS in this subgroup. These findings further corroborated the assumption that increased NT, even in the presence of a normal karyotype, may be an early sign of cardiac impairment or defect ${ }^{3-8,15-17}$ and that the underlying haemodynamic changes associated with TTTS may manifest as increased NT between 10-14 weeks of gestation. Chromosomopathies, with or without cardiac defects, were disclosed more frequently whenever increased NT was associated with abnormal flow in DV. ${ }^{3-8}$

This is not surprising when we take the sphincterlike ductus venosus as a crucial distributor of well oxygenated umbilical venous blood to the left atrium. In fact, DV appreciation as a regulatory shunt can add valuable information on foetal venous haemodynamics. Blood flow in the ductus is characterised by high velocity during ventricular systole (S-wave) and diastole (D-wave) and the presence of forward flow during atrial contraction (A-wave). In

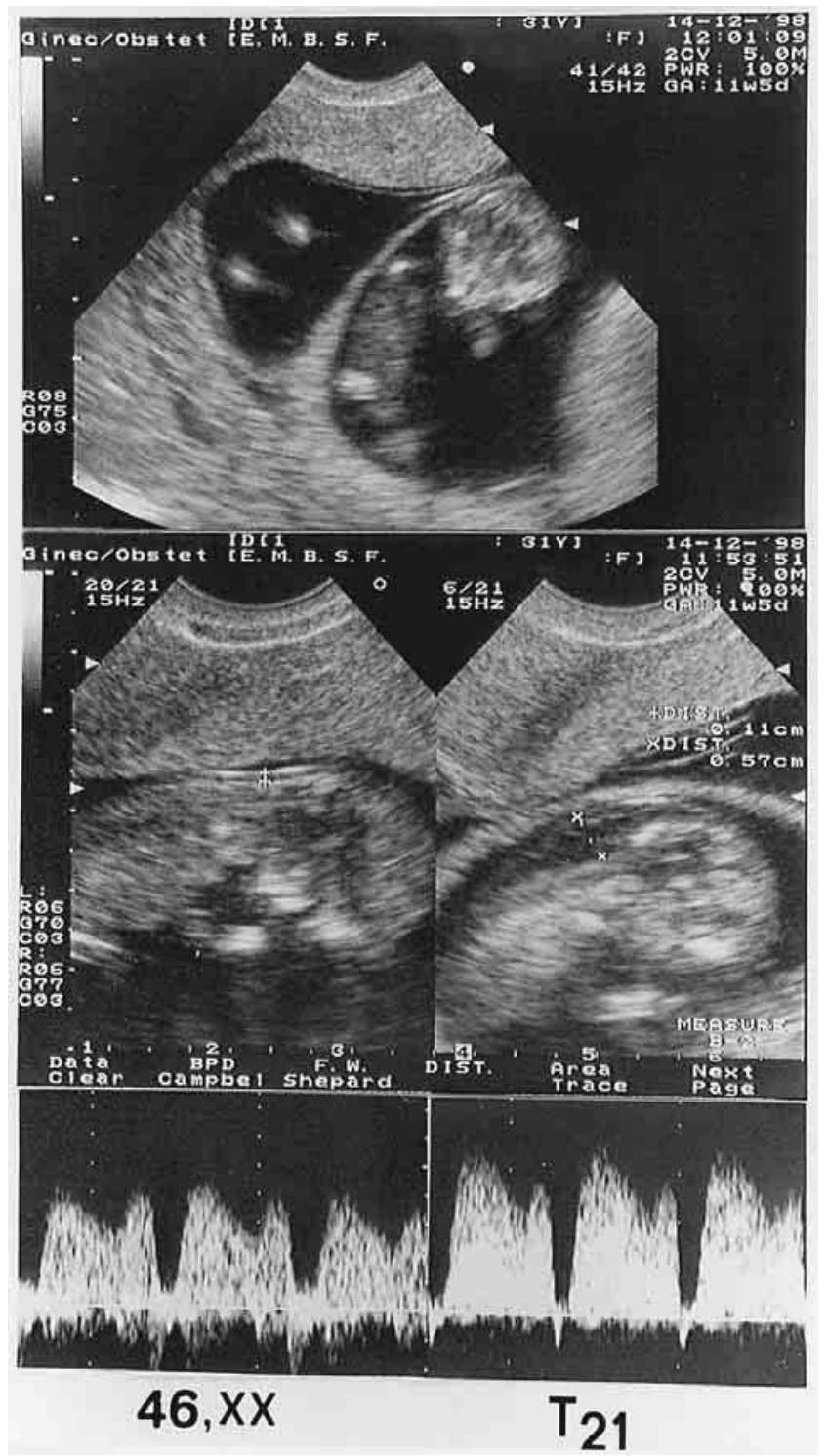

Figure 4 Dichorionic diamniotic twin pregnancy with nuchal translucency (NT) discrepancy at 11 weeks and 5 days (NT $=1.1 / 5.7 \mathrm{~mm}$ ). Doppler blood flow waveforms were obtained in both foetuses, showing a normal pattern in the foetus with normal NT $(46, X X)$ and a reverse flow during atrial contraction in the foetus with increased NT (trisomy 21)

contrast, in the normal pattern of both inferior vena cava and hepatic veins, with their low pressure, low velocity and compliant walls, there is reversed flow during atrial contraction. In cardiac failure, with or without cardiac defects, reversed flow during atrial contraction was also observed in the ductus venosus. $^{3-5,7,17}$

The most striking feature was the reduced or reversed velocity during atrial contraction commonly found in foetuses with congenital heart defects, ${ }^{5,7,17}$ growth retardation ${ }^{18}$ and TTTS. ${ }^{8,19}$ In all 
these clinical situations this particular haemodynamic alteration seems to reflect impaired cardiac performance and appears as a sign of ominous prognosis.

Specifically, in the first trimester of pregnancy, supportive evidence for heart strain or cardiac defect in foetuses with increased NT and anomalous venous return is provided by the findings of Hyett and colleagues. ${ }^{16,16}$ Recently they have shown augmented levels of mRNA for atrial natriuretic peptide in cardiac tissues from trisomic foetuses with increased NT thickness. Most recipient foetuses develop in utero a cardiac dysfunction in twin pregnancies complicated by TTTS. ${ }^{20}$ Foetuses with increased NT and anomalous flow in the DV presented more frequently a chromosomal abnormality, ${ }^{6,7}$ and even if chromosomally normal, a higher incidence of abnormalities of the heart and great arteries were documented. ${ }^{15}$ In this way it seems plausible that in monochorionic twin pregnancies increased NT in the recipient foetus may also be a manifestation of heart failure due to hypervolaemia. With advancing gestation, heart failure may resolve due to increased diuresis. Interestingly enough, foetuses developing severe TTTS Iater in pregnancy did not present sonographically detectable nuchal oedema.

The signs of highly pulsatile venous waveforms recorded in the foetus with increased NT in the first trimester of pregnancy are in good agreement with the haemodynamic al terations described by Hecher and co-workers ${ }^{19}$ in the recipient with fully established TTTS later in pregnancy. Umbilical vein pulsations correlated to atrial contraction and absent or reversed flow during atrial contraction in the DV are signs of congestive heart failure due to hypervolaemia and increased preload from placental vascular anastomotic transfusion. Zosmer et $\mathrm{al}^{20}$ proposed that cardiac dysfunction may be induced in utero by the sustained strain upon the heart by TTTS, predominantly affecting the right ventricle and, in fact, some surviving twins of TTTS show a persistent right ventricular cardiomiopathy.

In contrast, the significant reduction of blood flow velocity in umbilical artery recorded in the 'donor' is consistent with hypovolaemia and increased placental resistance, consecutive increase in cardiac afterload and decrease in umbilical venous return.

Another pertinent point of discussion is the safety of ultrasound bioeffects in such a vulnerable period as early pregnancy. If it is true that pulsed Doppler mode carries high energy output (but consistently less than the $100 \mathrm{~mW} / \mathrm{cm}^{2}$ considered safe by the competent entities), ${ }^{11,12}$ it is also true that the combined use of much lower energy output techniques, such as Colour Doppler and Power Doppler, will facilitate the identification of vascular struc- tures and shorten the time of foetal exposure to the ultrasound beam. Besides, care in using pulsed Doppler intermittently will ensure a short exposure time. Finally, bone ossification is incipient at this stage of pregnancy, reducing the danger of deleterious bioeffects.

Until now we could only diagnose a twin-twin transfusion syndrome in monochorionic pregnancies by identifying the disparity in foetal size and amniotic fluid volume between the donor and the recipient. It may well be that the presence of increased NT and abnormal venous return at 10-14 weeks of gestation in monochorionic twin pregnancies may provide an alarm signal of the subsequent development of TTTS. Both first trimester clues could anticipate the clinical features of a severe TTTS much earlier in the course of pregnancy and should motivate the ultrasonographer to undertake a closer surveillance of these twins. Therefore, it may well be that the better understanding of the underlying pathophysiology of cardiac dysfunction in TTTS may facilitate timely therapeutic strategies and thus improve the outcome of these high risk pregnancies.

\section{Acknowledgements}

We are indebted to Professor Kypros Nicolaides for the therapeutic approach in the two cases presented and the valuable discussion and suggestions on the present paper.

\section{References}

1 Sebire NJ, D'Ercole C, Hughes K, Carvalho M, Nicolaides KH. Increased nuchal translucency thickness at 10-14 weeks of gestation as a predictor of severe twin-to-twin transfusion syndrome. Ultrasound Obstet Gynecol 1997; 10: 86-89.

2 Cincotta RB, Fisk N. Current thoughts on twin-twin transfusion syndrome. Clin Obstet Gynecol 1997; 40(2): 290-302.

3 Montenegro N, Matias A, Areias JC, Castedo S, Barros H. Increased nuchal translucency: possible involvement of early cardiac failure. Ultrasound Obstet Gynecol 1997a; 10: 265-268

4 Matias A, Montenegro N, Areias $\mathrm{C}$, Brandão O. Anomalous venous return associated with major chromosomopathies in the late first trimester of pregnancy. Ultrasound Obstet Gynecol 1998; 11: 209-213.

5 Matias A, Gomes C, Flack N, Montenegro N, Nicolaides KH. Screening for chromosomal defects at 11-14 weeks: the role of ductus venosus blood flow. Ultrasound Obstet Gynecol 1998; 12: $380-384$.

6 Areias JC, Matias A, Montenegro N, Brandão O. Early antenatal diagnosis of cardiac defects using transvaginal Doppler ultrasound: new perspectives? Fetal Diagn Ther 1998; 13: 111-114. 
7 Matias A, Montenegro N, Areias JC, Brandão O. The importance of Doppler in the first trimester of gestation for the detection of fetal cardiac malformations. Adv Obstet Perinatol 1998; 9: 75-81.

8 Matias A, Montenegro N, Areias JC, Nicolaides KH. Anticipating twin-twin transfusion syndrome: the putative role of nuchal translucency and ductus venosus evaluation. Ultrasound Obstet Gynecol 1998; 8: 196 (abstract).

9 Hyett JA, Noble P, Snijders RMM, Montenegro N, Nicolaides $\mathrm{KH}$. Fetal heart rate in trisomy 21 and other chromosomal abnormalities at 10-14 weeks of gestation. Ultrasound Obstet Gynecol 1996; 7: 239-244.

10 Snijders R.M, Noble P, Sebire N, Souka A, Nicolaides KH. UK multicentre project on assessment of risk of trisomy 21 by maternal age and fetal nuchal translucency thickness at 10-14 weeks of gestation. Lancet 1998; 351: 343-346.

11 Food and Drug Administration. Guide for measuring and Reporting Acoustic Output of Diagnostic Ultrasound. Rockville, MD: FDA, Center for Devices and Radiological Health, 1992.

12 European Committee for UItrasound Radiation Safety. Clinical Safety Statement. Eur JUItrasound 1996; 4: 145.

13 Montenegro N, Matias A, A reias JC, Barros H. Ductus venosus revisited: a Doppler blood flow evaluation in the first trimester of pregnancy. Ultrasound Med Biol 1997; 23: 171-176.
14 Bebbington MW, Wittman BK. Fetal transfusion syndrome: antenatal factors affecting outcome. Am JObstet Gynecol 1989; 160: 913-915.

15 Hyett JA, Moscoso G, Papapanagiotou G, Perdu M, Nicolaides $\mathrm{KH}$. Abnormalities of the heart and great vessels in chromosomally normal fetuses with increased nuchal translucency thickness at 10-13 weeks of gestation. Ultrasound Obstet Gynecol 1996; 7: 245-250.

16 Hyett JA, Brizot ML, von Kai senberg C, McKie AT, Farzenah F, Nicolaides KH. Cardiac gene expression of atrial natriuretic factor and brain natriuretic peptide in trisomic fetuses. Obstet Gynecol 1996; 87: 506-510.

17 Kiserud T, Eik-Nes SH, Hellevik LR, Blaas HG. Ductus venosus blood vel ocity changes in fetal cardiac diseases. JMatern Fetal Invest 1993; 3: 15-20.

18 Kiserud T, Eik-Nes SH, Blaas HG, Hellevik LR, Simensen B. Ductus venosus blood velocity and the umbilical circulation in the seriously growth retarded fetus. Ultrasound Obstet Gynecol 1994; 4: 109-114.

19 Hecher K, Ville Y, Snijders R, Nicolaides KH. Doppler studies of the fetal circulation in twin-to-twin transfusion syndrome. Ultrasound Obstet Gynecol 1995; 5: 318-324.

20 Zosmer N, Bajoria R, Weiner E, Rigby M, Vaughan J, Fisk N. Clinical and ecographic features of in utero cardiac dysfunction in the recipient twin-to-twin transfusion syndrome. $\mathrm{Br}$ Heart J 1994; 72: 74-79. 